\title{
Traduire
}

Ine autre perspective sur la tataduction

Revue française de la traduction

$244 \mid 2021$

Des jeux et des mots

\section{Bien traduire les jeux de société. L'art délicat de jongler entre traduction technique et littéraire}

\author{
Maïlys Lejosne-Le Calvez
}

\section{(2) OpenEdition}

Édition électronique

URL : https://journals.openedition.org/traduire/2330

DOI : $10.4000 /$ traduire.2330

ISSN : 2272-9992

Éditeur

Société française des traducteurs

Édition imprimée

Date de publication : 15 juin 2021

Pagination : 65-72

ISSN : 0395-773X

\section{Référence électronique}

Maïlys Lejosne-Le Calvez, «Bien traduire les jeux de société. L'art délicat de jongler entre traduction technique et littéraire », Traduire [En ligne], 244 | 2021, mis en ligne le 15 juin 2021, consulté le 01 juillet 2021. URL : http://journals.openedition.org/traduire/2330 ; DOI : https://doi.org/10.4000/traduire. 2330 


\section{Bien traduire les jeux de société}

L'art délicat de jongler entre traduction technique et littéraire

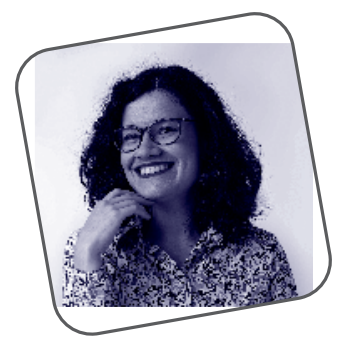

Maillys Lejosne-Le Calvez

\section{Introduction}

La traduction des jeux de plateau est un marché de niche en pleine expansion. II s'agit d'une traduction spécialisée qui nécessite une bonne connaissance du monde du jeu et de ses codes, mais aussi une grande rigueur d'exécution. En effet, les jeux de société dits «modernes» se sont enrichis et largement diversifiés ces dernières années. On est très loin désormais du Uno, du Monopoly ou du Scrabble, même si ces derniers continuent à apparaître en tête des ventes. Plus de 1000 jeux sortent tous les ans en France, premier marché européen devant l'Allemagne et le Royaume-Uni. Le jeu de société représentait un chiffre d'affaires de 328 millions d'euros en 2019 et des ventes supérieures à 19 millions de boîtes selon une étude NPD Group. Les prévisions indiquent par ailleurs une tendance à la hausse pour 2020 en raison du confinement.

Les acteurs du marché sont multiples, entre maisons d'édition, distributeurs, créateurs. Ces derniers ont souvent recours au financement participatif via Kickstarter ou Ulule, ou encore via des plateformes dédiées au jeu de société 
comme les Polonais de Gamefound ou la française Game On Tabletop. De nombreuses maisons d'édition leur ont emboîté le pas et emploient elles-mêmes ce type de plateforme pour financer leurs projets souvent multilingues.

On est donc face à un marché "de niche» où se croisent traducteurs internes, freelance et amateurs passionnés, parfois bénévoles. Cependant, la complexification du marché et son essor nécessitent une professionnalisation de toute la chaine et la prise au sérieux de la traduction comme partie intégrante du succès d'un jeu. En effet, traduire correctement les composantes d'un jeu et le cas échéant les localiser est un aspect essentiel de son succès sur d'autres marchés. II n'y a rien de pire qu'une règle mal traduite qu'on n'aura pas envie de lire, ou qu'un manque de cohérence dans la terminologie qui embrovillera les joveurs. On est donc bien face à une traduction technique spécialisée. Mais est-ce uniquement cela?

\section{De la traduction technique, vraiment?}

Traduire une règle de jeu peut techniquement s'apparenter à traduire un manuel utilisateur. Les règles doivent être didactiques, compréhensibles, faciles à suivre, adaptées à l'âge du public, au type de joveur et à son pays de destination. Une règle mal écrite nuira au plaisir du jeu. Personne n'a envie de passer trois heures à déchiffrer une règle ou d'interrompre le jeu toutes les cing minutes pour s'y reporter...

On n'emploiera pas le même vocabulaire ou niveau de langue dans un jeu à partir de 7 ans ou à partir de 14 ans. De même, on n'utilisera pas le même vocabulaire pour un jeu dit «familial» ou visant des joueurs occasionnels, ou pour un jeu dit «expert», destiné à des joveurs aguerris. Un jeu d'ambiance est par définition un jeu dont les règles doivent être rapidement assimilables et courtes. Ce sont d'ailleurs souvent des boîtes de petit format avec des livrets très brefs, voire simplement des dépliants en accordéon à deux ou trois plis. Parfois même, le livret de règles comporte plusieurs langues pour faciliter la production et la distribution dans plusieurs pays. Il faut alors faire preuve de concision, surtout quand le coefficient de foisonnement entre la langue source et la ou les langues cibles est positif. 
Il faut aussi connaître son public francophone. Ainsi, les Québécois brassent les cartes et les dés, tandis que les Français mélangent ou battent les cartes et jettent ou lancent les dés.

Le jeu "expert», quant à lui, demandera encore plus de rigueur en ce qui concerne la terminologie spécialisée. II n'est pas question de confondre le game turn et le game round par exemple, ni les jetons, les tuiles, les pions ou encore les meeples (terme désormais consacré pour parler des pions en bois représentant des personnages et qui serait une contraction de my people datant de 2000). Le recours à un outil de traduction assistée par ordinateur (TAO) peut dans ce cas s'avérer utile, voire crucial pour s'assurer de la cohérence de la terminologie et de la phraséologie. Le respect de cette dernière est indispensable pour que le lecteur joueur puisse s'y retrouver dans le type d'instructions et d'actions qu'on lui explique.

De plus, chaque type de jeu a sa propre terminologie et son propre jargon. Les jeux de figurines vont par exemple recourir à des expressions différentes si on parle de jeu d'escarmouche ou de jeu d'affrontement (wargames).

La mise en page et la logique d'enchaînement de la règle diffèrent aussi selon les pays. Ainsi, l'ordre des chapitres d'une règle écrite à l'américaine pourra sembler abscons et illogique à un public français. D'un côté, la règle est organisée comme suit: présentation de l'univers, description du contenu de la boîte, détails des éléments du jeu, tour de jeu, conditions de victoire. À l'inverse, en France, on commence par les conditions de victoire, puis on privilégie l'explication du tour de jeu, avant l'explication détaillée des différents éléments et actions. Cela explique qu'un lecteur non habitué à la structure logique doive feuilleter tout le livret plusieurs fois à la recherche d'un point particulier (surtout si ce livret contient une vingtaine de pages sans table des matières). Dans ce cas précis, la comparaison avec un manuel utilisateur prend tout son sens. Il pourra donc parfois s'avérer nécessaire, pour une meilleure lisibilité, compréhension et assimilation, de réorganiser des chapitres, de rajouter un sommaire ou un glossaire, voire d'adapter le glossaire existant (parfois appelé livret de référence et constituant un livret à part entière distinct du livre de règles). 
C'est sans compter sur les jeux inspirés d'œuvres culturelles qui demandent une recherche documentaire importante pour respecter la terminologie et la phraséologie existantes. Les jeux dits «à licence» n'hésitent pas à citer des extraits des œuvres dont ils sont tirés, auquel cas il faut aller récupérer la traduction originale, qu'il s'agisse d'un roman, d'un film ou de tout autre support. Pour ne citer qu'un exemple parmi tant d'autres, l'œuvre d'H. P. Lovecraft, tombée dans le domaine public en 2008 et à la terminologie précise et particulière, a donné lieu à de nombreuses adaptations ludiques sous des formes diverses et variées. L'époque du jeu a aussi un impact sur le champ lexical à utiliser et à respecter, car celui-ci varie grandement selon qu'il s'agit d'un jeu historique ou futuriste par exemple. Ainsi, on peut avoir à traduire un extrait de journal du XIXe siècle pour un jeu d'enquête ou un jeu de rôle. Cela rajoute une composante littéraire à l'aspect technique.

\section{La traduction de jeu de société: une traduction littéraire?}

Après avoir passé en revue les différents aspects techniques de la traduction de jeu de société, nous allons maintenant aborder ses aspects littéraires. Ces derniers varient considérablement d'un jeu à l'autre en fonction du type de jeu et du public visé.

On peut ainsi retrouver un aspect littéraire, ou plutôt créatif, dans certains jeux d'ambiance, notamment dans la localisation de jeux de mots, d'énigmes ou de références culturelles, mais ce n'est pas le type de jeu où cet aspect ressortira le plus.

Les composantes littéraires sont en effet plus nombreuses dans les jeux à forte tendance narrative. Le jeu de rôle en est un parfait exemple. Celui-ci a connu un regain ces dernières années après une baisse d'intérêt due à un effet de diabolisation en France dans les années 1990. L'élément principal d'un jeu de rôle est souvent son livre de base qui présente aussi bien la mécanique de jeu que l'univers et généralement un scénario d'initiation. Outre les règles qui nécessitent une traduction technique rigoureuse, ce livre comporte aussi une partie fortement littéraire qu'on appelle fluff dans le jargon. II s'agit de tout ce qui ne constitue pas l'explication technique d'une règle: la mise en contexte du jeu, la description de 
l'univers, des décors... Le fluff peut également inclure des dialogues, des bandes dessinées, des poèmes, éventuellement une nouvelle, voire des jeux de mots ou encore des énigmes qui demandent un vrai travail de localisation et d'adaptation non seulement à la culture, mais aussi au public cible.

Le fluff se retrouve aussi dans les jeux narratifs, les jeux d'enquête, certains jeux de figurines... Cette partie demande très clairement une bonne créativité et une maîtrise de l'univers imaginé et de ses codes. Un background de jeu de rôle se lit comme un roman et demandera des ressources créatives pour inventer des néologismes et localiser des noms propres ou communs. C'est notamment le cas dans les univers fantastiques (qu'ils soient de fantasy ou de science-fiction) qui usent et abusent de néologismes. II n'est pas rare dans ce cas-là de traduire des noms de personnages, de villes, de pays, de pouvoirs magiques... Cependant, comme abordé précédemment, nombre de jeux de rôle sont aussi tirés d'œuvres diverses (livres, films, séries télévisées...). Il faudra donc faire preuve de créativité tout en veillant à respecter la terminologie et la phraséologie des traductions existantes.

Mais il ne faut pas oublier non plus les scénarios qui mêlent traduction technique aussi bien que littéraire. Ils comportent d'une part l'arbre de décisions et l'enchaînement logique des différentes scènes auxquelles participent leurs personnages joveurs et d'autre part des textes d'ambiance, de décor, des aides de jeu avec une forte composante littéraire.

\section{De l'homogénéité des supports}

Nous avons jusqu'ici beaucoup parlé de la traduction des livres de règles; cependant, traduire un jeu de société ne se résume pas à cela. Il faut parfois traduire de nombreux supports: des cartes, pour les jeux de deckbuilding par exemple, des plateaux, d'éventuels codex, des livrets de références, des livrets d'apprentissage, des aides de jeu, les boîtes de jeu elles-mêmes, voire une application mobile. Quid alors de l'homogénéité?

Prenons le cas de l'application mobile dont l'utilisation se développe de plus en plus ces dernières années. II n'est pas rare que la personne responsable de la traduction de l'application mobile soit différente de celle se chargeant des autres 
composantes du jeu. II s'avère alors primordial d'avoir un glossaire établi et de maintenir l'homogénéité de la terminologie sur tous les supports. Imaginez que l'application vous indique de récupérer une carte, mais que le titre de cette dernière n'a pas été traduit de façon identique sur les supports numérique et physique. Vous devez alors passer en revue tout le paquet en vous appuyant, dans le meilleur des cas, uniquement sur son illustration pour la retrouver. La relecture de l'ensemble est dans ce cas cruciale pour s'assurer de la cohérence terminologique globale.

Dans le cas d'une production multilingue, les différents supports peuvent arriver à des dates échelonnées en fonction du planning de relecture et de validation dans la langue source. Ainsi, les cartes arriveront parfois plusieurs semaines avant le livret de règles ou vice versa. Il faudra donc encore une fois faire attention à maintenir la cohérence et l'homogénéité, surtout là encore si ce n'est pas la même équipe qui travaille sur les différents éléments du projet.

\section{La mise en page, un vrai casse-tête}

La traduction d'un jeu ne peut pas être décorrélée de sa présentation. Qu'il s'agisse d'un livre de règles ou de cartes, la mise en page fait partie intégrante de la traduction. Le document source inclut bien souvent des icônes dans le corps même du texte ou des illustrations légendées. Peut-on alors avoir recours uniquement à un outil de TAO? Doit-on intégrer la mise en page dans notre prestation globale? C'est une question qui mérite d'être posée. En effet, il n'est pas rare que les supports arrivent directement au format InDesign et que le client final demande une prestation de publication assistée par ordinateur (PAO) en plus de la traduction ou de la révision. Le fait de pouvoir modifier soi-même les fichiers mis en page est un avantage certain quand l'espace est extrêmement contraint. Ainsi, c'est beaucoup plus simple de faire preuve de concision quand on a visuellement la mise en page sous les yeux que depuis son outil de TAO. En effet, les livres de règles regorgent d'encadrés, d'illustrations légendées, d'exemples de jeu commentés... Par conséquent, travailler sur la traduction uniquement dans un outil de TAO ou simplement à partir d'une extraction du texte en fichier Word 
s'avère extrêmement compliqué et peut entraîner des oublis, voire des problèmes de continuité ou de cohérence.

Le cas des icônes dans le corps du texte est intéressant. On voit souvent apparaitre au fil du texte des icônes présentes sur des cartes ou des plateaux. Voici un exemple:

«Les représentent les points de vie de votre personnage.»

J'ai utilisé ici un caractère existant dans la police (un cœur), mais la plupart du temps, il s'agira d'une illustration, c'est-àdire d'un objet en PAO. Si le fichier texte est mal exporté, par exemple à partir d'un fichier PDF, les illustrations ne seront pas reconnues et n'apparaîtront pas dans le corps du texte sous quelque forme que ce soit. Cela peut entraîner des anomalies, des mots manquants et des problèmes de mise en page finale. En effet, si l'objet n'est pas ancré correctement dans le bloc texte approprié au sein du logiciel de PAO, toute modification de mise en page entraînera un décalage par rapport au texte, ce qui pourra, dans les cas extrêmes, nuire à la lisibilité et à la compréhension. Il revient donc à la personne qui traduit d'être consciente de ce genre de problématique. Plusieurs solutions sont bien sûr envisageables, comme mettre en place un système de codification en attribuant un caractère spécial ou un code à chaque objet différent dans le texte à traduire; cela permet aussi à la personne chargée de la PAO de savoir quels remplacements effectuer. Mais cela nécessite une excellente préparation de la traduction, qui anticipe ces difficultés. Tous ces aspects doivent être pris en compte lors de la gestion de projet pour permettre une bonne coordination de tous les intervenants.

Recourir à un traducteur ou une traductrice maitrisant les outils de PAO s'avère alors un avantage certain pour avoir une vue d'ensemble du projet, éviter certains écueils inhérents à la traduction de jeux de société et obtenir un produit final à la hauteur des attentes.

\section{Conclusion}

Joueuse moi-même, j'ai essayé d'être le plus exhaustive possible tout en restant concise. J'espère que cet article vous aura permis de découvrir un domaine à part entière extrêmement riche et varié. En effet, même si celui-ci peut 
paraître frivole par son aspect ludique, une mauvaise traduction peut ruiner une expérience de jeu. Les règles sont le premier élément auquel on s'intéresse quand on découvre pour la première fois un jeu, c'est donc la première impression qu'il laisse. Des règles mal comprises seront indubitablement mal traduites et cela nuira à l'expérience ludique du public cible.

La traduction de jeu de société requiert donc de maîtriser non seulement la traduction technique, mais aussi la traduction littéraire, sans oublier la mise en page. Cependant, pour bien traduire une règle de jeu, il faut aussi la comprendre et en saisir la mécanique, ce qui implique la nécessité de jover soi-même et de s'être confronté à la multiplicité des jeux existants sur le marché.

\section{contact@fae-traduction.fr}

Maillys Lejosne-Le Calvez est traductrice et interprète de liaison depuis 2017. Elle fonde, en 2018, FAE Traduction dont le credo est «Donner des ailes à vos mots». Ses langues de travail sont l'anglais, l'espagnol et le français. Ingénieure procédés de formation (HEl, 2009), elle obtient en 2019 son diplôme de niveau 7 de traduction technique (Edvenn) en parallèle au lancement de son activité. Elle aime endosser de multiples casquettes. Outre son activité de traductrice pour l'industrie et le domaine ludique, elle est aussi formatrice ou encore démonstratrice de jeux sur les salons spécialisés.

SOURCES CITÉES

TUTT Frédérique, Les Français se prennent aujeu, France, NPD Group, https://www. npdgroup.fr/wps/portal/npd/fr/actu/communiques-de-presse/les-francais-seprennent-au-jeu/, consulté le 11/03/2021.

COCHRANE, Guylaine, «Le foisonnement, phénomène complexe», in TTR, 8 (2), 1995, p. 175-193, https://doi.org/10.7202/037222ar, consulté le 28/04/2021. 